\title{
HOMOGENEOUS PRIMARY ABELIAN GROUPS
}

\section{DALTON TARWATER}

A primary abelian group will be said to be homogeneous if it is either divisible or a direct sum of cyclic groups of a fixed order $p^{n}$. Kaplansky [1, p. 66] states that if $G$ is a homogeneous primary group, then for any two subgroups $S$ and $T$, there is an automorphism of $G$ carrying $S$ into $T$ if and only if $S$ and $T$ are isomorphic, and $G / S$ and $G / T$ are isomorphic. It is the purpose of this paper to show that homogeneous primary groups are characterized by a property which is not far removed from Kaplansky's statement. Finally, a counterexample to Kaplansky's statement will be provided.

Throughout the paper all groups will be $p$-primary abelian groups. $G\left[p^{n}\right]$ will denote, as usual, the subgroup of elements $x$ of $G$ such that $p^{n} x=0$.

THEOREM. $G$ is a homogeneous p-group if and only if for all subgroups $S$ and $T$ of $G$ the two conditions are equivalent:

(a) There is an automorphism of $G$ sending $S$ onto $T$.

(b) $S$ and $T$ are isomorphic and $G[p] / S[p]$ is isomorphic to $G[p] / T[p]$.

Proof. Since $G[p]$ is a characteristic subgroup of any primary group $G$, it is always the case that (a) implies (b). Suppose then that $G$ is homogeneous and $S$ and $T$ are subgroups of $G$ satisfying condition (b). If $G$ is divisible, let $E$ and $E^{\prime}$ be injective hulls of $S$ and $T$, respectively, in $G$. Then $S[p]=E[p], T[p]=E^{\prime}[p]$, and $G=E \oplus H=E^{\prime} \oplus K$ for some divisible subgroups $H$ and $K$ of $G$. Any isomorphism between $S$ and $T$ extends to an isomorphism between $E$ and $E^{\prime}$. Furthermore,

$$
H[p] \cong G[p] / E[p]=G[p] / S[p] \cong G[p] / T[p] \cong K[p],
$$

and since $H$ and $K$ are divisible it follows that $H$ and $K$ are isomorphic. Hence any isomorphism of $S$ into $T$ extends to an automorphism of $G$.

If, on the other hand, $G$ is a direct sum of cyclic groups of fixed order $p^{n}$, then $G$ can be imbedded in its injective hull $D$ so that $G[p]$ $=D[p]$ and $G=D\left[p^{n}\right]$. Now $S$ and $T$ satisfy (b) in $D$, which is divisible; hence any isomorphism between $S$ and $T$ extends to an automorphism of $D$. Since $G=D\left[p^{n}\right]$ is a characteristic subgroup of $D$, this automorphism induces an automorphism of $G$. Hence if $G$ is homogeneous, conditions (a) and (b) are equivalent.

Received by the editors April 7, 1969. 
To prove the converse, suppose $G$ is a primary abelian group with the required property. Let $x$ and $y$ be any two elements in $G[p]$, and let $S$ be the cyclic subgroup generated by $x$ and $T$ that generated by $y$. Then $S$ and $T$ are isomorphic, as are $G[p] / S[p]$ and $G[p] / T[p]$. Hence there is an automorphism of $G$ sending $S$ onto $T$. It follows that $x$ and $y$ have the same height in $G$. Thus all elements of $G[p]$ have the same height in $G$. If this common height is infinite, $G$ is divisible; otherwise, $G$ is a direct sum of cyclics of the same order $p^{n}$. $G$ is therefore seen to be homogeneous and the proof is complete.

REMARK. It is easy to see that the theorem could be restated: A necessary and sufficient condition for a primary group $G$ to be homogeneous is that for each pair of isomorphic subgroups $S$ and $T$ of $G$ such that $G[p] / S[p]$ is isomorphic to $G[p] / T[p]$, each isomorphism of $S$ and $T$ is induced by an automorphism of $G$.

COROLlaRy. Let $G$ and $H$ be isomorphic homogeneous groups with isomorphic subgroups $S$ and $T$ respectively. Each isomorphism of $S$ onto $T$ extends to an isomorphism of $G$ onto $H$ if and only if $G[p] / S[p]$ is isomorphic to $H[p] / T[p]$.

The example which was promised earlier is obtained by taking $G=Z\left(p^{\infty}\right) \oplus G^{\prime}$, where $G^{\prime}$ is divisible of infinite rank, $S=G[p]$ and $T=G^{\prime}[p]$. Then $S$ and $T$ are isomorphic, as are $G / S$ and $G / T$. But no automorphism sends $S$ into $T$. Conversely, if $G$ and $S$ are as above and $T=G$, then each automorphism of $G$ sends $S$ into $T$, while $G / S$ and $G / T$ are not isomorphic.

\section{REFERENCE}

1. I. Kaplansky, Infinite abelian groups, Univ. of Michigan Press, Ann Arbor, Mich., 1954. MR 16, 444.

Texas Technological College 